Gynäkologe 2018 · 51:692

https://doi.org/10.1007/s00129-018-4311-8

(c) Springer Medizin Verlag GmbH, ein Teil von Springer Nature 2018

CrossMark

\author{
Hans Ludwig' · K. Diedrich ${ }^{2}$ \\ 'Basel, Schweiz \\ ${ }^{2}$ Groß Sarau, Deutschland
}

\title{
Das Jubiläum einer Fachzeitschrift: Der Gynäkologe wird 50
}

Als die Gründer, E.J. Plotz, O. Käser und V. Friedberg, 1967 übereinkamen, eine neue Zeitschrift für die Fortbildung im Fach Frauenheilkunde ins Leben zu rufen und der Springer-Verlag seine Bereitschaft erkennen ließ, das Projekt zu unterstützen und für Ausstattung und Vertrieb zu sorgen, waren alle Beteiligten zufrieden, bald über ein neues wissenschaftliches Organ zu verfügen. Im Jahr 1968 begann das Projekt, Wirklichkeit $\mathrm{zu}$ werden. Es fand sich gut ein in das Konzert der schon bestehenden Publikationen, die Fachzeitschrift Archiv für Gynäkologie und die jüngere Geburtshilfe und Frauenheilkunde wurden nun ergänzt. Niemand konnte damals jedoch wissen, wie gut das neue Medium in die bekannte Wissenslandschaft hineinpassen würde. Bald zeigte sich aber, dass es ein Erfolg wurde. In einigen Jahren war man so weit, dass man von der vierteljährlichen Erscheinungsweise auf eine 2-monatliche, schließlich auf eine monatliche Verfügbarkeit übergehen konnte. Die Frauenärzte und Frauenärztinnen abonnierten zunehmend häufig und begannen zu schätzen, was ihnen damit geboten wurde.

) Niemand konnte 1968 wissen, wie gut sich Der Gynäkologe in die Wissenschaftslandschaft einfügen würde

Die Qualität hing von denen ab, welche die Beiträge schrieben. Den vielen Ausgaben in dem vergangenen halben Jahrhundert ist anzusehen, wie groß die Mühe war, die sich die meisten gaben, wenn sie aufgefordert wurden, über ihr jeweiliges Gebiet zusammenfassend zu schreiben. In regelmäßiger Folge wurden perinatalmedizinische, onkologische, endokrinologische und fortpflanzungsmedizinische Bereiche aufgegriffen. Jede Innovation wurde sehr bald akzentuiert. Das Autorenverzeichnis liest sich auch rückblickend wie eine Personengeschichte des Fachs. Im Laufe der Jahre gab es niemanden, der sich einen Namen in der klinischen Forschung der deutschsprachigen Länder gemacht hatte, der nicht dann und wann mit einem Beitrag auftauchte. Einige Namen kehrten sogar mehrmals in jedem Jahr wieder, andere fielen dadurch auf, dass sie wissenschaftliche Trends zutreffend vorherzusagen wussten oder Kontroversen, gelegentlich auch Fehlentwicklungen aufzeichneten. Der Leser gewann so Einsichten, die ihm allein aus der täglichen ärztlichen Arbeit verborgen geblieben wären. Offenbar freute man sich auf jede neue Ausgabe, erst recht dann, als die Rubriken an Zahl zunahmen und neben den 3 klassischen Säulen des Fachs auch sozialmedizinische, juristische, ethische, berufspolitische und historische Fragen behandelt wurden. So wurde Der Gynäkologe vielfach zu einer Art Selbstverständlichkeit, wenn man sich mit dem gegenwärtigen Stand des Wissens oder Könnens im Fach vertraut machen wollte.

Wir wünschen uns, dass die Zeitschrift auch die nächsten Jahre über lebendig bleibt, keine Kompromisse in der Qualität eingeht und auch fachliche Probleme oder Sorgen aufgreift, bevor solche überhand nehmen oder gar bedrohlich werden können.

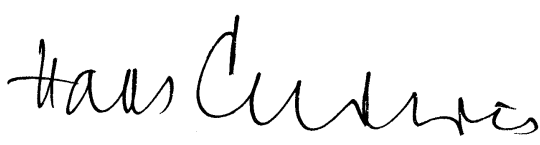

Hans Ludwig, Basel

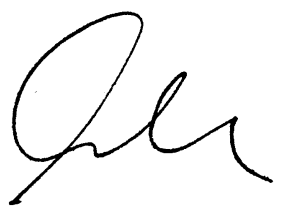

Klaus Diedrich, Hamburg

\section{Korrespondenzadresse}

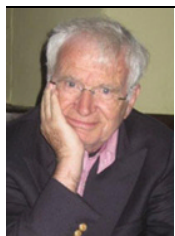

Prof. em. Dr. med. H. Ludwig, FRCOG, FACOG hon.

Gellertstr. 137, 4052 Basel, Schweiz prof.ludwig@bluewin.ch

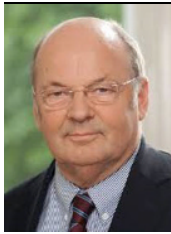

Prof. em. Dr. med. Dr. h.c. mult. K. Diedrich

Klein Sarau 45, 23627 Groß Sarau, Deutschland prof.diedrich@gmail.com

Interessenkonflikt. H. Ludwig und K. Diedrich geben an, dass kein Interessenkonflikt besteht. 